\title{
Non-LTE Model Spectra for Gaseous Planetary Debris Disks around WDs
}

\author{
Stephan Hartmann, Thorsten Nagel, Thomas Rauch \\ and Klaus Werner
}

Institute for Astronomy and Astrophysics, University of Tübingen, Germany email: hartmann@astro.uni-tuebingen.de

\begin{abstract}
Metal-rich dust disks around white dwarfs are thought to be the debris of tidally disrupted rocky bodies. While normally the number of features to study the planetary material directly is very limited, ancillary gas disks around some of these white dwarfs provide the opportunity to do so. We used our Tübingen Accretion Disk code ACDC, assuming non-LTE conditions, to model the gaseous spectrum component. We investigated the chemical mixture as well as the surface density and effective temperature, and utilized the Ca II infrared triplet to determine the geometry of the disk.
\end{abstract}

Keywords. accretion disks, (stars:) white dwarfs, (stars:) planetary systems, line: profiles, radiative transfer

Analyses of ultraviolet and optical spectra of white dwarfs (WDs) revealed a large number of metal-rich single objects. The picture of the WDs' atmospheres getting polluted due to the accretion of planetary material, e.g. tidally shredded planetesimals, became quite successful in explaining these observations (Farihi, Jura \& Zuckerman 2009). Usually the almost homogeneous $\mathrm{H}$ or He atmospheres are used as a detector for the spectral signatures of the pollution, as the debris disks themselves, consisting of cold, dusty material yield only few features to analyze the accreted planetary material. However, about $20 \%$ of these dusty disks are connected to an additional gaseous disk of similar radial extent (Melis et al. 2010). The hotter gas becomes noticeable through double peaked emission lines which provide the unique opportunity to directly analyze the debris material before it is accreted onto the WD. Hallmark to the gaseous disk component is the CaII $\lambda \lambda$ 8498, 8542, $8662 \AA$ infrared triplet (IRT), which often shows prominent asymmetric line profiles (Gänsicke et al. 2006)

The Tübingen Accretion Disk code AcDC (Nagel et al. 2004) calculates the spectrum of an axisymmetric, gaseous disk applying the well known $\alpha$-disk method (Shakura \& Sunyaev 1973). The disk is described in form of a set of discrete rings, for which vertical structure calculations can be performed independently from each other. Assuming nonlocal thermodynamic equilibrium conditions, the radiative transport, the equations of hydrostatic and energetic equilibrium, and the rate equations are solved simultaneously. During the final surface-integration step over the ring spectra, the Keplerian rotation is taken into account which results in double-peak line profiles.

To model the gaseous component of a metal-rich debris disk, we include $\mathrm{H}, \mathrm{C}, \mathrm{O}$, $\mathrm{Mg}, \mathrm{Si}, \mathrm{S}$, and $\mathrm{Ca}$ with abundances similar to a solar system asteroid-like mixture into our models $(\mathrm{C}=2.82 \%, \mathrm{O}=48.08 \%, \mathrm{Mg}=15.33 \%, \mathrm{Si}=19.80 \%, \mathrm{~S}=11.50 \%$, and $\mathrm{Ca}=2.48 \%$, mass fractions). We calculated the disks in a non-stationary scheme using different surface density $\Sigma$ and effective temperature $T_{\text {eff }}$ values. The disk's radial extent can be varied by the number and radii of rings used to simulate the radial structure. 

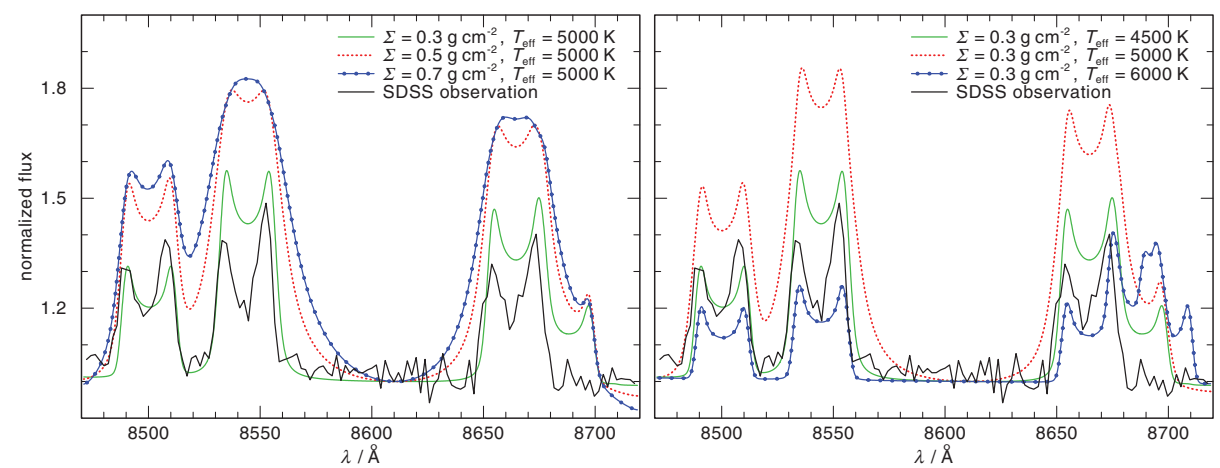

Figure 1. Ca II IRT of non-LTE disk models for three different values of $\Sigma$ (left) and $T_{\text {eff }}$ (right) compared with the observation of SDSS J122859.93+104032.9.
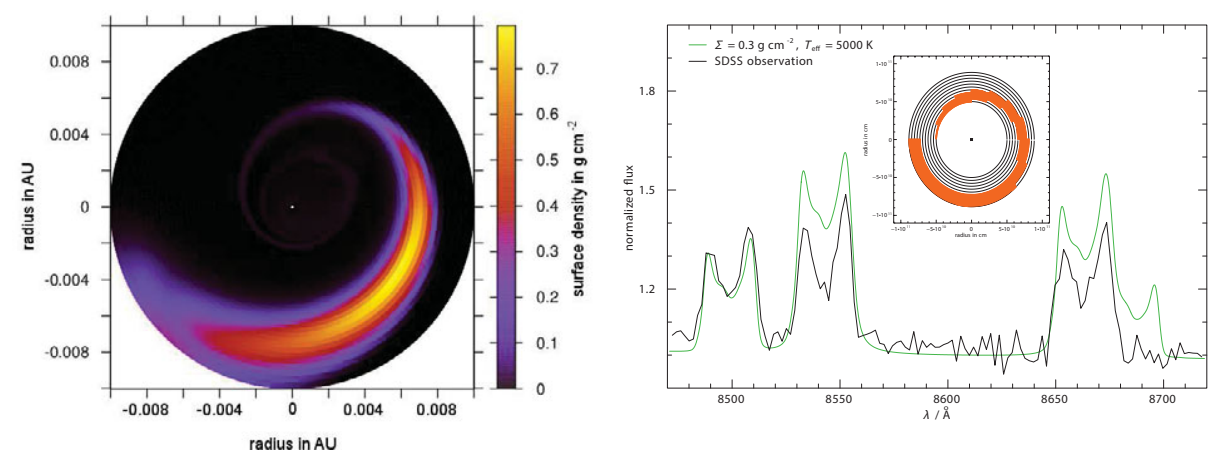

Figure 2. Left: A hydrodynamic calculation shows an asymmetric gas disk. Right: Modeled spectrum for a similar non-axisymmetric disk geometry (inlaid picture).

Disk spectra for radial constant values of $\Sigma$ and $T_{\text {eff }}$ were calculated to investigate the influence on the Ca II IRT (Fig. 1). Higher $\Sigma$ values tend to broaden the lines and therefore reduce the dip between the lines' double peaks. Raising $T_{\text {eff }}$ results in more similar line strengths of all three components. We compare the models to the observations of SDSS J122859.93+104032.9, the first WD hosting a gaseous debris disk reported (Gänsicke et al. 2006), achieving our best fit (green, solid line in all figures) for $\Sigma=0.3 \mathrm{~g} \mathrm{~cm}^{-2}$ and $T_{\text {eff }}=5000 \mathrm{~K}$.

While for these models a circular disk is assumed, hydrodynamic simulations using FARGO (Masset 2000) suggest that a tidally disrupted rocky body would form a more asymmetric disk (Fig. 2, left panel). By modifying the surface-integration step of ACDC to use non-axisymmetric geometries, we are able to model the observed asymmetry of the Ca II IRT line profiles (Fig. 2, right panel). Further details of our analysis approach were described in Hartmann et al. (2011).

\section{References}

Farihi, J., Jura, M., \& Zuckerman, B. 2009, ApJ, 694, 805

Gänsicke, B. T., Marsh, T. R., Southworth, J., et al. 2006, Science, 314, 1908

Hartmann, S., Nagel, T., Rauch, T., et al. 2011, A\& $A$, 530, A7

Masset, F. 2000, A\&AS, 141, 165

Melis, C., Jura M., Albert L., et al. 2010, ApJ, 722, 1078

Nagel, T., Dreizler, S., Rauch, T., et al. 2004, AESA, 428, 109

Shakura, N. I. \& Sunyaev, R. A. 1973, A\&A, 24, 337 